\title{
Prevalence and Factors Associated with Meconium Staining of Amniotic Fluid, Meconium Aspiration Syndrome and Clear Liquor in South Indian New-born-Part II
}

\author{
Grace Lalana Christopher \\ Grace Specialist Clinic, \#6, 1st Floor, Maruthi Complex, Ramamurthynagar Main Road, Bangalore 560016, South India.
}

How to cite this paper: Grace Lalana Christopher. (2020) Prevalence and Factors Associated with Meconium Staining of Amniotic Fluid, Meconium Aspiration Syndrome and Clear Liquor in South Indian New-born-Part II. International Journal of Clinical and Experimental Medicine Research, 4(4), 126-137.

DOI: 10.26855/ijcemr.2020.10.003

Received: July 6, 2020

Accepted: July 28, 2020

Published: August 13, 2020

*Corresponding author: Grace Lalana Christopher, Grace Specialist Clinic, \#6, 1st Floor, Maruthi Complex, Ramamurthy Nagar Main Road, Bangalore 560016, South India.

Email: drlalana@gmail.com

\begin{abstract}
Meconium staining of amniotic fluid (MSAF) is a commonly observed phenomenon in day-to-day practice of obstetrics, advocates the quick delivery of baby as priority since majority $84 \%$ of newborns with MSAF were asphyxiated at birth OR 8.42 [CI95\%] 5.1-14, P=0.0001 compared to those newborns with clear liquor. The prevalence of MSAF 7.8\% however incidence of Meconium Aspiration Syndrome (MAS) was 1.8\%, but constituted 23\% of MSAF parturient. Various high risk factors include primigravida, young mothers with obstetric complications of premature rupture of membranes (PROM) of more than one hour before labor occurred in 9.1\% of 207MSAF pregnancies compared to only $7.3 \%$ in overall births. Similarly also Pregnancy induced hypertension (PIH) occurred in 9\% with MSAF compared to an overall 5.6\%. Gestational diabetes (GDM) was least 3.4\% in MSAF pregnancies compared to overall 5.3\%. While Grade II MSAF peaked in birth weight category 300-3499g category, most newborn of remaining parturient weighed between 2,500-2,999g. Peak births at 38 weeks comprised of newborns with clear liquor delivered normally, contrasted to peak births at 39 weeks for newborns with MSAF and MAS delivered mainly by emergency Lower Segment Caesarean Section (LSCS). Surprisingly commonest parturient blood group was $\mathrm{AB}+\mathrm{ve}$. In conclusion, effective preventive strategy would be early delivery by 38 weeks gestation preferably by elective LSCS, obviating intra-partum events in high risk cases, resulting in improvement of fetal and neonatal morbidity and mortality.
\end{abstract}

\section{Keywords}

Meconium Stained Amniotic Fluid, Meconium Aspiration Syndrome, Clear Liquor, Parturient Blood Group, Preventive Strategy

\section{Introduction}

Meconium staining of amniotic fluid been reported in 8\%-20\% of all deliveries [1-5]. Aspiration and may occur antepartum, intrapartum or even at infant's first breath, however its significance is as a sign of fetal distress, indicating an asphyxial event in fetus that results in-utero gasping with aspiration of meconium and its complications $[6,7]$. Meconium aspiration syndrome (MAS) occurs worldwide in about $2 \%-9 \%$ neonates, often results in severe mental retardation and cerebral palsy, accounting for $12 \%$ of neonatal mortality and $2 \%$ of perinatal mortality rate 
[7]. Transient umbilical cord compression during contractions triggers foetal hypoxic stress causing vagal stimulation, resulting in mesenteric vasoconstriction, increased peristalsis and relaxation of anal sphincter leading to intrauterine passage of meconium, stimulating gasping movements that result in meconium aspiration [8]. Fetuses that have passed meconium during labor are in the state of temporary compensated fetal distress with peripheral hypoxia but well-oxygenated vital organs. At this stage, the fetal blood $\mathrm{pH}$ will not show acidemia and Apgar score will be good if delivered soon [6,9]. Late deceleration of fetal heart rate (FHR) pattern, most commonly associated with lower fetal scalp blood $\mathrm{pH}<7.25$ indicate a state of decompensated fetal distress in which quick intervention becomes necessary [10-12].

The extent of lung destruction in MAS with obstruction of both large and small airways depends on degree of hypoxia and acidosis present at birth as well as volume and more so the concentration of the meconium aspirated [13]. Fetal lungs normally contain, alveolar liquid about $40 \mathrm{ml}$ of body weight, differs from amniotic fluid or plasma ultra-filtrate having low $25 \mathrm{mg} / \mathrm{dl}$ protein, but there is reduced production of pulmonary fluid in fetal hypoxemia with acidotic foetus and meconium inhibits foetal lung fluid reabsorption at birth disturbing the ability of lungs to adapt to extra uterine life $[13,14]$. Continuous positive pressure ventilation (CPPV) with oxygen in both MSAF and MAS new-borns with birth asphyxia facilitates the reabsorption of lung fluid into interstitial, thence to lymphatic, triggering smooth complex fetal cardiovascular changes adapting to adult life $[14,15]$.

MAS on X-ray chest may show coarse, patchy infiltrates radiating from the hilum into the peripheral lung fields with focal areas of atelectasis, consolidation, or emphysema [16]. Histopathology show meconium with squamous epithelial cells and yellow to brown granular amorphous material deposited in alveoli and bronchioles with focal alveolar haemorrhages and evidence of bronchopneumonia but no organism can be cultured from these lung specimens, except in preterm fetus, meconium passage usually occurs with infections such as Listeria monocytogenesis, Ureaplasma urealyticum, and rotavirus which may cause fetal enteritis [17, 18]. However, meconium concentrations exceeding $1 \%$ enhances the ability of amniotic fluid to sustain bacterial growth by inhibition of neutrophil phagocytic activity [19].

Hence, it is fetal hypoxia in MAS that enhances the potential for poor outcome, as it is the antepartum chronic or acute hypoxial damage to the lungs that causes persistent pulmonary hypertension, or else it is mild, benign and self-limiting [20]. The partial or complete airway obstruction in MAS leads to hyperinflation with surrounding atelectasis and increased lung resistance that decreases lung compliance with resultant acute hypoxemia, hypercapnia, respiratory acidosis, pulmonary vasoconstriction, persistent pulmonary hypertension, ventilation perfusion mismatch and right to left shunting of blood through the foramen ovale and patent ductus arteriosus [16, 21, 22], with subsequent asphyxiated fetal death or distressed neonates [7, 12, 23]. The incidence of respiratory failure, diagnosed by $\mathrm{PO}_{2} 50 \mathrm{~mm}$ of $\mathrm{Hg}, \mathrm{PCO}_{2} 75 \mathrm{~mm}$ of $\mathrm{Hg}$ or repeated or prolonged episodes of apnoea and circulatory failure occurs in $53 \%$ in MAS with neonatal mortality of $20 \%$, necessitating prompt intervention and delivery with MSAF [7, 23].

\section{Aim}

The retrospective study was undertaken:

* To estimate prevalence of MSAF, subdivided into significant and non-significant MSAF as well incidence of newborns with MAS and clear liquor;

* To determine association of birth asphyxia in newborns with MSAF compared to those with clear liquor;

* To compare various biological risk factors of new-born with MSAF, MAS and clear liquor;

* To estimate distribution of parturient blood group compared to general population;

* To identify preventive strategy to improve fetal and neonatal outcome in MSAF and MAS.

\section{Materials and Methods}

The study comprised 2,750 consecutive singleton live births during the period January $1^{\text {st }} 2015$ to May $31^{\text {st }}$ 2017 at Shifa Hospital, a multispecialty centre in the metropolitan city of Bangalore. There were 207 cases with MSAF and 49 cases of MAS. The exclusion criteria were 22 twin delivery and 46 stillbirths. Consistency of meconium was graded as non-significant Grade I, meconium staining being thin, light yellow-green meconium stained amniotic fluid, and significant Grade II, meconium staining defined as thick, dark green, viscous with particles or 'pea soup'. Meconium Aspiration Syndrome (MAS) is defined as presence thick, dark green, viscous MSAF at birth followed subsequently by sign and symptoms of MAS (i.e., tachypnoea, dyspnoea, retraction, grunting or cyanosis). 
Simultaneous biological risk factors such as parity, mother's age, and obstetric complications, mode of delivery, birth weight and gestation of newborns with MSAF, MAS and Clear Liquor were compared and strategy determined as effective preventive measures for MSAF in reducing birth asphyxia, thereby promoting fetal and neonatal wellbeing.

All women received adequate prenatal care, gestation was assessed by Last Menstrual Period (LMP) and correlated with ultrasound measurement of Crown-rump length (CRL) undertaken within 18 to 20 weeks of gestation to determine fetal age. If CRL and menstrual dates agreed to within the normal range of error ( \pm 7 days) days then LMP was used to establish fetal age. Birth weight was recorded within 24 hours on a digital weighing machine accurate up to $\pm 10 \mathrm{~g}$. Sources of data were Labor room register, new born register and neonatal records. Data was entered into EPI data version 3.1 and then exported to SPSS Version 21 for analysis of distribution statistics like frequencies and cross tabulation and statistical significance was performed.

\section{Results}

During the study period 207 newborns with MSAF, constituted 7.5\% of singleton live births, seen in Table 1. A majority 83.6\% ( $\mathrm{n}=173$ ) of newborns had MSAF and were asphyxiated at birth with OR 8.42 [CI95\%] 5.1-14 being highly statistically significant $\mathrm{P}=0.0001$, being 30 times at higher risk as compared to $92.45 \%$ ( $\mathrm{n}=2543$ ) controls with clear liquor that constituted only $16 \%$ birth asphyxia cases. In contrast, all $100 \%$ of newborns who developed MAS were asphyxiated at birth.

Table 1. Frequency of Meconium staining of amniotic fluid

\begin{tabular}{ccc}
\hline Group & No. of cases & Percent \\
\hline Clear Liquor & 2,543 & 92.47 \\
MSAF & 207 & 7.52 \\
\hline
\end{tabular}

Two groups with MSAF were graded based on the consistency of meconium as thin or thick. Grade I, non-significant, thin meconium stained amniotic fluid and Grade II, significant, thick meconium or particulate matter at spontaneous or artificial rupture of membranes. Of 207 MSAF cases, Grade I or thin meconium staining of amniotic fluid constituted 68.1\% ( $\mathrm{n}=141)$ while Grade II with thick meconium staining comprised 31.9\% $(\mathrm{n}=66)$ seen in Table 2.

Table 2. Severity of Meconium staining

\begin{tabular}{ccc}
\hline Groups & No. of cases & Percent \\
\hline Thin MSAF & 141 & 68.1 \\
Thick MSAF & 66 & 31.9 \\
& 207 & 100 \\
\hline
\end{tabular}

While 2,543 cases with clear liquor comprised 92.5\% of 2,750 live births, 207 MSAF cases with Grade I or thin meconium staining constituted 5.1\% ( $\mathrm{n}=141 / 2750)$ ) and Grade II, thick meconium staining was a low $2.4 \%$ $(n=66 / 2750)$ ), the frequency and type of Meconium seen in Table 3.

Table 3. Frequency and type of Amniotic Fluid

\begin{tabular}{ccc}
\hline Group & No. of cases & Percent \\
\hline Clear Liquor & 2,543 & 92.5 \\
Thin MSAF & 141 & 5.1 \\
Thick MSAF & 66 & 2.4 \\
\hline
\end{tabular}

Newborns with MSAF who did not develop MAS constituted 5.7\% $(n=158 / 2750)$ births seen in Table 4. The incidence of Meconium Aspiration Syndrome (MAS) was 1.9\% ( $\mathrm{n}=49 / 207)$ with sign and symptoms of respiratory distress such as tachypnoea, dyspnoea, retraction, grunting or cyanosis after birth but constituted $24 \%$ $(\mathrm{n}=49 / 207)$ new-borns with MSAF seen in Table 5.

Table 4. Frequency of Meconium Aspiration Syndrome (MAS)

\begin{tabular}{ccc}
\hline Group & No. of cases & Percent \\
\hline Clear Liquor & 2,543 & 92.5 \\
Thin MSAF & 158 & 5.7 \\
MAS & 49 & 1.9 \\
& 2,750 & 100 \\
\hline
\end{tabular}


Table 5. Meconium Aspiration Syndrome among MSAF cases

\begin{tabular}{ccc}
\hline Group & No. of cases & Percent \\
\hline MSAF without MAS & 158 & 76.3 \\
MAS & 49 & 23.7 \\
& 207 & 100 \\
\hline
\end{tabular}

\section{Distribution by Gravida}

Grade I MSAF occurred in 50.4\% (n=70/139) primigravida similarly also Grade II MSAF with 50.6\% (n=29/57) as also MAS with $46 \%(n=21 / 46)$. In contrast, 31\% $(n=793 / 2,518)$ newborns with clear liquor were primigravida with almost similar distribution of $30 \%(n=764)$ for second gravid followed by $22 \%(n=551 / 2,518)$ third gravida mothers, distribution seen in Figure 1 and Table 6.

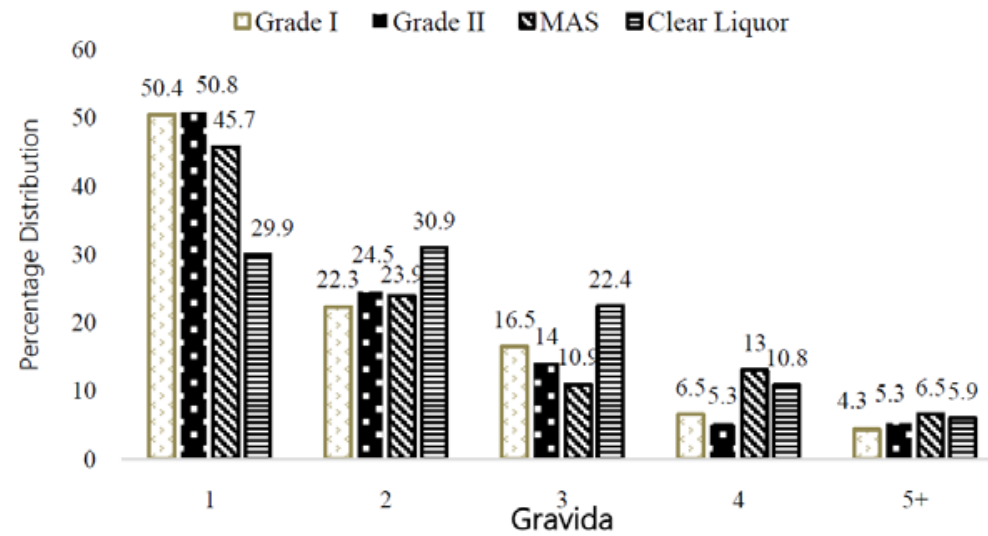

Figure 1. Percentage distribution of newborn with Grade I \& II MSAF, MAS and Clear Liquor according to Gravida.

Table 6. Distribution by Gravida

\begin{tabular}{cccccccc}
\hline & \multicolumn{7}{c}{ Gravida } \\
\hline Groups & 1 & 2 & 3 & 4 & $5+$ & No. & Missing \\
Grade I & 70 & 31 & 23 & 9 & 6 & 139 & 2 \\
Grade II & 29 & 14 & 8 & 3 & 3 & 57 & 9 \\
MAS & 21 & 11 & 5 & 6 & 3 & 46 & 3 \\
Clear Liquor & 793 & 764 & 551 & 262 & 148 & 2,518 & 21 \\
\hline
\end{tabular}

\subsection{Distribution by Mother's Age}

Grade I MSAF newborns were mostly 44\% (n=6/139) were born to mothers aged 20-24 years, similarly also $42 \%(n=25 / 60)$ with Grade II MSAF. However, newborns with MAS peaked at $45 \%(n=20 / 46)$ in older mothers aged 25-29 years. Newborns with clear liquor majority 39\% $(n=940 / 2,413)$ were born to young mother's aged 20-24 years, distribution seen in Figure 2 and Table 7.

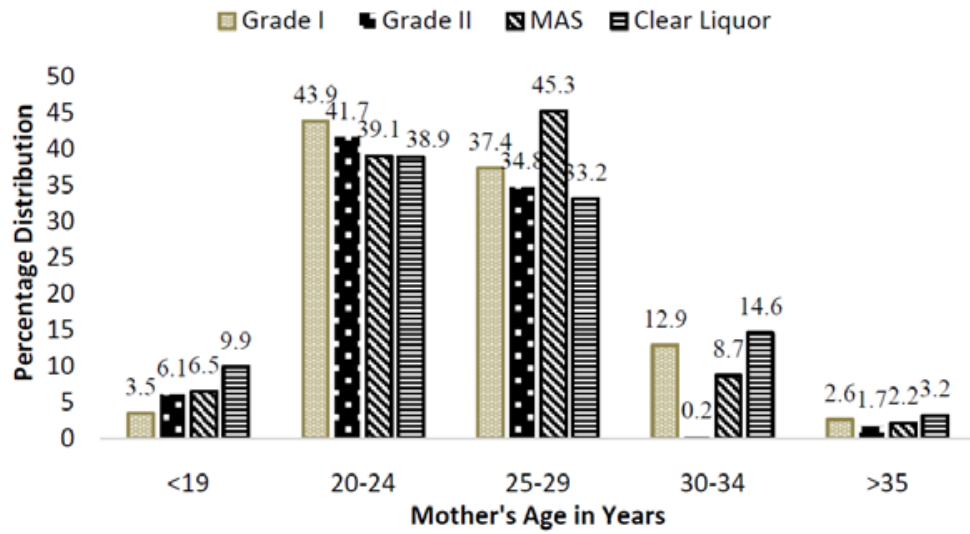

Figure 2. Percentage distribution of Grade I \& II MSAF, MAS and Clear Liquor according to Mother's Age. 
Table 7. Distribution by Mother's Age

\begin{tabular}{cccccccc}
\hline & \multicolumn{7}{c}{ Mother's Age in Years } \\
\hline Groups & $<19$ & $20-24$ & $25-29$ & $30-34$ & $>35$ & No. & Missing \\
Grade I & 5 & 61 & 52 & 18 & 3 & 139 & 2 \\
Grade II & 4 & 25 & 23 & 7 & 1 & 60 & 6 \\
MAS & 3 & 18 & 20 & 4 & 1 & 46 & 3 \\
Clear Liquor & 240 & 940 & 803 & 352 & 78 & 2,413 & 126 \\
\hline
\end{tabular}

\subsection{Distribution by Obstetric Complications}

Most 78.3\% ( $n=162)$ of 207 pregnancies with MSAF had no obstetric complications while $22 \%(n=45)$ had obstetric complications seen in Table 8. Premature rupture of membranes (PROM) of more than one hour before labor occurred in $9.1 \%$ of 207 pregnancies with MSAF compared to only $7.3 \%$ in overall births. Similarly also Pregnancy induced hypertension (PIH) occurred in 9\% with MSAF compared to an overall 5.6\%. Gestational diabetes (GDM) was least 3.4\% in MSAF pregnancies compared to overall 5.3\%.

Table 8. Obstetric Complications with Meconium staining of amniotic fluid

\begin{tabular}{ccc}
\hline Obstetric Complications & Number of cases & Percentage \\
\hline Nil Obstetric complications & 162 & 78.3 \\
Obstetric complications & 45 & 21.7 \\
Total & 207 & 100 \\
\hline
\end{tabular}

While PROM was commonest in pregnancies with Grade II MSAF 57\% (n=8/14), followed by Grade I MSAF35\% ( $n=11 / 31)$ and newborns with clear liquor peaked 40\% ( $\mathrm{n}=181 / 457)$ with PROM. In contrast PIH occurred maximum with MAS 57\% ( $n=4 / 7)$ followed by Grade I MSAF 52\% ( $n=16 / 31)$. GDM was least 13\% $(n=4 / 31)$ with Grade I MSAF to maximum 43\% $(n=3 / 7)$ in MAS and Grade II MSAF $21 \%(n=3 / 14)$. Newborns with clear liquor showed almost equal 30\% distribution for PIH and GDM, distribution seen in Figure 3 and Table 9.

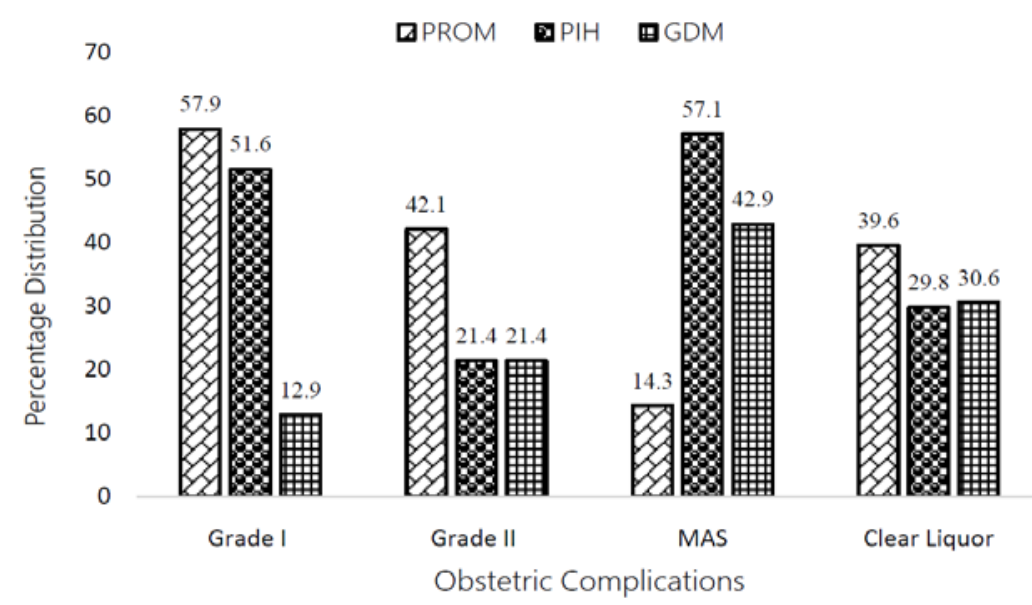

Figure 3. Percentage distribution of New-born with Complications of pregnancy in Grade I, II MSAF, MAS and Clear Liquor.

Table 9. Obstetric Complications associated with MSAF, MAS and Clear Liquor

\begin{tabular}{ccccc}
\hline & \multicolumn{2}{c}{ Obstetric Complications } & & No. \\
Groups & PROM & PIH & GDM & 31 \\
Grade I & 11 & 16 & 3 & 14 \\
Grade II & 8 & 3 & 3 & 7 \\
MAS & 1 & 4 & 140 & 457 \\
Clear Liquor & 181 & 136 & & 4 \\
\hline
\end{tabular}




\subsection{Distribution by Birth weight}

Most 38\% ( $\mathrm{n}=53 / 138)$ of newborns with Grade I thin MSAF weighed between 2,500-2,999g with 34\% ( $n=47 / 138)$ weighing 3,000-3,499g. However, Grade II thick MSAF peaked 30\% $(n=20 / 152)$ in birth weight group of 3,000-3,499g, similarly also MAS peaked 31\% ( $n=14 / 45)$ with equal distribution $29 \%(n=13 / 45)$ in 2,500-2,999g and 2,000-2,499g with minimal or none in lower birth weights. In contrast newborns with clear liquor peaked 38\% (n=902/2,342) in 2,500-2,999g birth weight category followed by 35\% $(\mathrm{n}=808)$ in 3,000-3,499g birth weight and 15\% (348) in 2,000-2,499g, distribution seen in Figure 4 and Table 10.

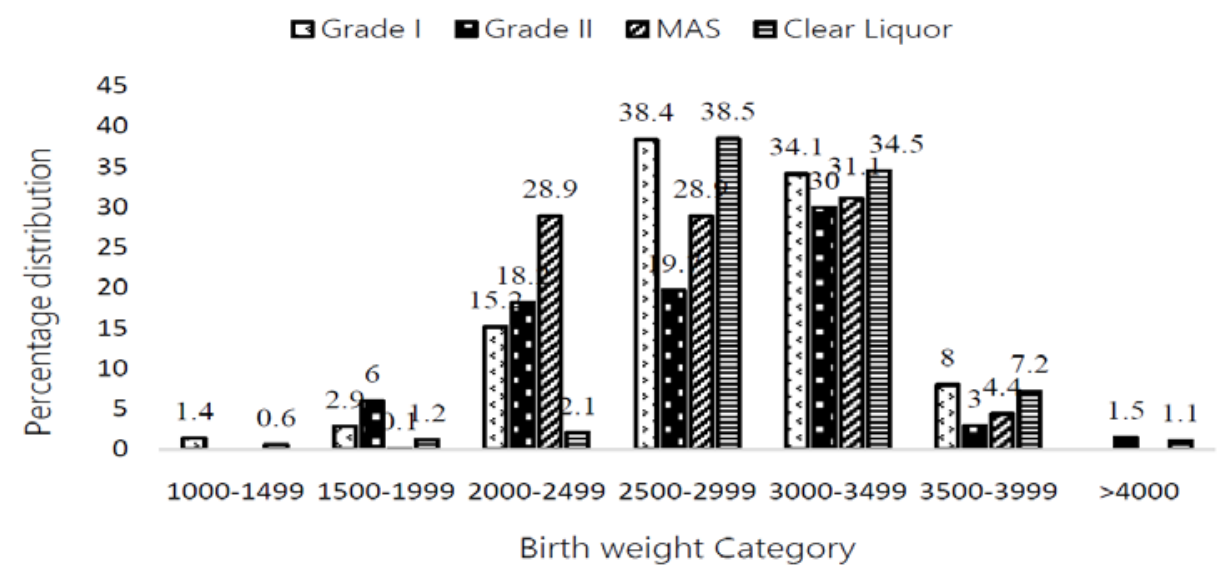

Figure 4. Percentage Distribution of New-borns by birth weight in Grade I \& II MSAF, MAS and Clear Liquor.

Table 10. Distribution by Birth weight category

\begin{tabular}{ccccccccccc}
\hline \multicolumn{10}{c}{ Birth weight in grams } \\
\hline Groups & $\leq 999$ & $1,000-1,499$ & $1,500-1,999$ & $2,000-2,499$ & $2,500-2,999$ & $3,000-3,499$ & $3,500-3,999$ & $>4,000$ & No. & Missing \\
Grade I & - & 2 & 4 & 21 & 53 & 47 & 11 & - & 138 & 3 \\
Grade II & - & - & 4 & 12 & 13 & 20 & 2 & 1 & 52 & 14 \\
MAS & - & - & 3 & 13 & 13 & 14 & 2 & - & 45 & 4 \\
Clear Liquor & 16 & 27 & 46 & 348 & 902 & 808 & 169 & 26 & 2,342 & 201 \\
\hline
\end{tabular}

\subsection{Distribution by Gestation}

Grade II thick MSAF peaked 38\% (n=22/58) with birth at 39 weeks gestation followed by 35\% ( $\mathrm{n}=16 / 46)$ newborns with MAS as well as Grade I thin MSAF 32\% ( $\mathrm{n}=43 / 133)$ at 39 weeks gestation. However, peak 27\% $(n=663 / 2,467)$ newborns with clear liquor were born at 38 weeks gestation with $26.6 \%(n=658 / 2,467)$ at 39 weeks gestation, reveals increased risk of MAS and Grade I and II MSAF for births at 39 weeks and beyond as compared to 38 weeks gestation or below, distribution seen in Figure 5 and Table 11.

aGrade I Grade II EMAS 目Clear Liquor

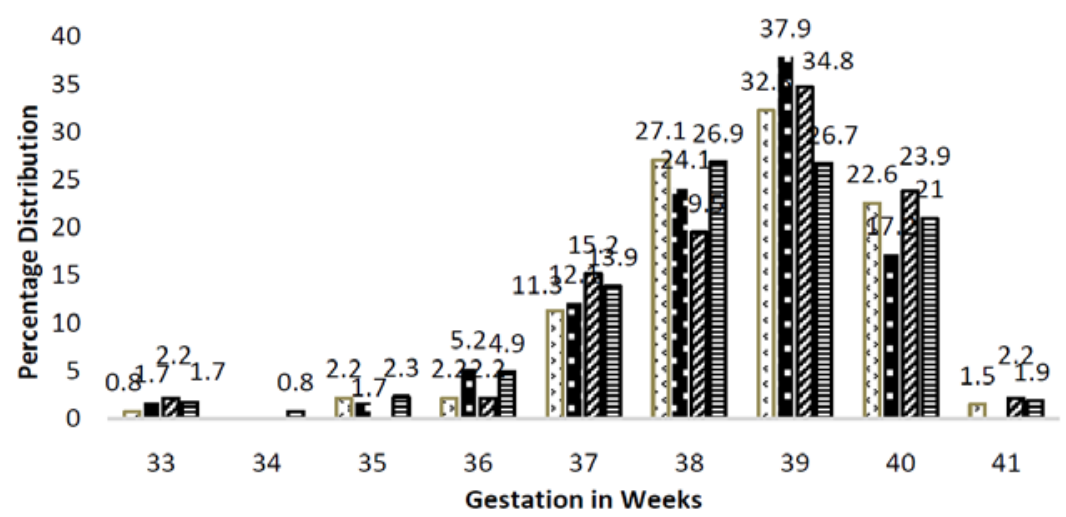

Figure 5. Percentage Distribution of New-borns with Grade I \& II MSAF, MAS and Clear Liquor according to Gestation. 
Table 11. Distribution by Gestation

\begin{tabular}{cccccccccccc}
\hline & \multicolumn{10}{c}{ Gestation in Weeks } \\
\hline Groups & 33 & 34 & 35 & 36 & 37 & 38 & 39 & 40 & 41 & No. & Missing \\
Grade I & 1 & & 3 & 3 & 15 & 36 & 43 & 30 & 2 & 133 & 8 \\
Grade II & 1 & & 1 & 3 & 7 & 14 & 22 & 10 & & 58 & 8 \\
MAS & 1 & & & 1 & 7 & 9 & 16 & 11 & 1 & 46 & 3 \\
Clear Liquor & 42 & 20 & 57 & 121 & 344 & 663 & 658 & 518 & 44 & 2,467 & 72 \\
\hline
\end{tabular}

\subsection{Distribution by Mode of Delivery}

According to mode of delivery, majority 54\% ( $n=1,378 / 2,532)$ normal vaginal births had clear liquor. In contrast, in MAS peaked 52\% $(n=24 / 47)$, Grade II thick MSAF 51\% $(n=28 / 55)$ and Grade I thin MSAF 47\% $(n=65 / 139)$ were delivered by emergency LSCS with common indication being fetal distress, had high risk of birth asphyxia, OR 4.91, [CI 95\%] 3.94-6.10 times normal delivery, being highly statistically significant $\mathrm{P}=0.0001$ being 83\% in MSAF cases. A comparatively low one-fourth incidence 26\% (n=662/2532) with clear liquor were delivered by emergency LSCS. However, newborns delivered by elective section had low risk of birth asphyxia, OR 1.67 [CI 95\%] 0.84-1.63, but statistically insignificant $\mathrm{P}=0.358$, most had clear liquor $15 \%$ ( $\mathrm{n}=388 / 2532)$ with less than 8\% with Grade I, II MSAF and MAS. Majority of newborns with vacuum extraction suffered high incidence of MAS associated with a significant higher risk of birth asphyxia, OR 8 [CI 95\%] 5.58-11.69, being highly statistically significant $\mathrm{P}=0.0001$. Quick intervention by emergency section, indicated by fetal distress in MSAF probably reduced severity of MAS with most clinical features resolving in 2-3 days' time, distribution seen in Figure 6 and Table 12.

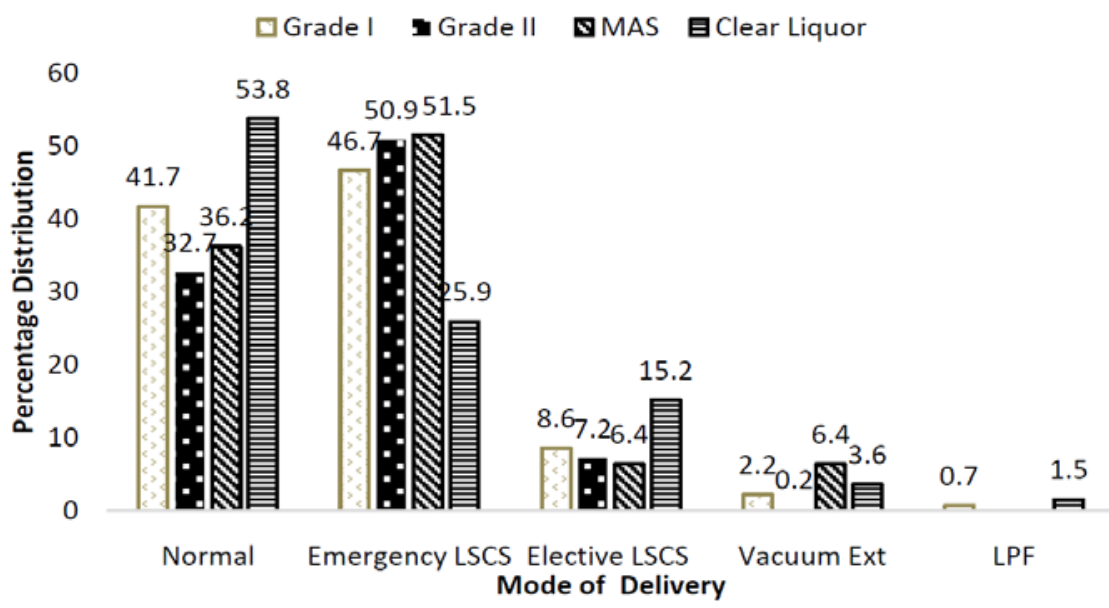

Figure 6. Percentage Distribution of New-borns with Grade I \& II MSAF, MAS and Clear Liquor according to Mode of delivery.

Table 12. Distribution by Mode of Delivery

\begin{tabular}{cccccccc}
\hline & \multicolumn{9}{c}{ Mode of Delivery } \\
Groups & Normal & Emergency LSCS & Elective LSCS & Vacuum Ext & LPF & No. & Missing \\
\hline Grade I & 58 & 65 & 12 & 3 & 1 & 139 & 2 \\
Grade II & 18 & 28 & 4 & 5 & - & 55 & 11 \\
MAS & 17 & 24 & 3 & 3 & - & 47 & 1 \\
Clear Liquor & 1,376 & 662 & 388 & 91 & 39 & 2,532 & 7 \\
\hline
\end{tabular}

\subsection{Distribution of Parturient Blood Group}

The commonest blood group was $\mathrm{AB}+\mathrm{ve}$, incidence 33\% ( $\mathrm{n}=774 / 2283)$ parturient, followed by $31 \%$, $\mathrm{A}+\mathrm{ve}$ $(n=706 / 2283)$, and $O+v e 23 \%(n=528 / 2283)$, distribution seen in Table 13 . Most $56 \%(n=23 / 41)$ of cases with MAS had mothers with AB+ve blood group, while 39\% $(n=50 / 127)$ of mothers with Grade I MSAF as also 33\% 
( $n=/ 2081$ ) newborns with clear liquor had AB+ve blood grouping. While O+ve occurred among 24\% (n=30/127) in Grade I MSAF as well as with clear liquor $24 \%(n=488 / 2081)$. In contrast, Grade II MSAF had mainly 34\% $(n=21 / 61)$ parturient with B-ve followed $21 \%(n=13 / 61)$ with blood groups of $A+v e$ and $A B-v e$, distribution seen in Figure 7 and Table 14.

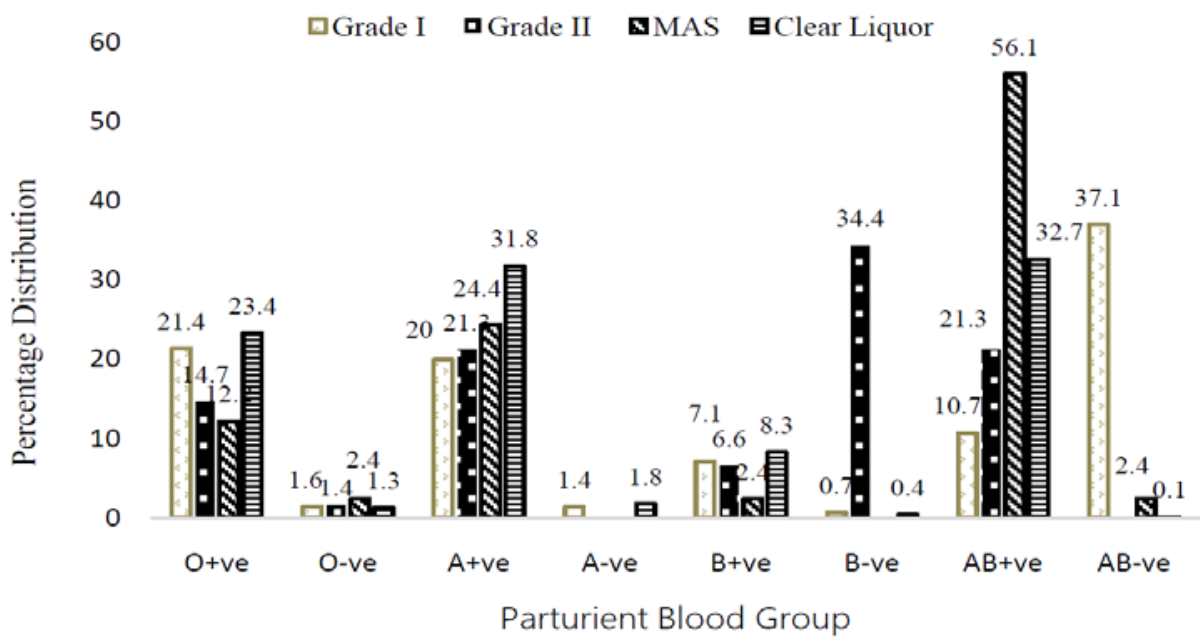

Figure 7. Percentage distribution of Parturient Blood Group with Grade I \& II MSAF, MAS and Clear Liquor.

Table 13. Number and Percentage distribution of Parturient by Blood Group

\begin{tabular}{ccccccccc}
\hline \multicolumn{10}{c}{ Parturient Blood Group } \\
\hline Overall & O+ve & O-ve & A+ve & A-ve & B+ve & B-ve & AB+ve & AB-ve \\
Number & 528 & 30 & 706 & 40 & 187 & 10 & 744 & 38 \\
Percent & 23 & 1 & 31 & 2 & 8 & .1 & 33 & 2 \\
\hline
\end{tabular}

Table 14. Distribution by Blood Group among Parturient with MSAF, MAS and Clear Liquor

\begin{tabular}{ccccccccccc}
\hline \multicolumn{10}{c}{ Parturient Blood Group } \\
\hline Groups & O+ve & O-ve & A+ve & A-ve & B+ve & B-ve & AB+ve & AB-ve & No. & Missing \\
Grade I & 30 & 2 & 28 & 2 & 10 & 1 & 50 & 4 & 127 & 109 \\
Grade II & 9 & 1 & 13 & - & 4 & 21 & 13 & - & 61 & 5 \\
MAS & 5 & 1 & 10 & - & 1 & - & 23 & 1 & 41 & 8 \\
Clear Liquor & 488 & 27 & 662 & 38 & 173 & 9 & 682 & 2 & 2,081 & 458 \\
\hline
\end{tabular}

\section{Discussion}

Meconium is a germ-free thick black green odorless material first recognized in fetal intestine around $10^{\text {th }}$ to $16^{\text {th }}$ week of gestation, stored in colon containing gastrointestinal and pancreatic secretions, bile, bile acids, mucus, cellular debri and swallowed vernixcaseosa and lanugo. Meconium is usually passed 24-48 hours after birth, while meconium passage in utero is a normal physiological event in term fetus and not a sign of fetal distress in the absence of fetal heart rate abnormalities [24, 25]. MSAF is present in $8 \%-20 \%$ of all deliveries increasing to $23 \%-52 \%$ after 42 weeks of gestation and rarely found in amniotic fluid before 34 weeks gestation, with about 21\% MSAF, developing MAS, one-third of these requiring intubation and mechanical ventilation [1-6, 23, 24]. In the present study, prevalence of MSAF was $7.5 \%$ and MAS $1.8 \%$ but which constituted $23 \%$ of MSAF cases. Grading of MSAF revealed 68\% thin MSAF and 32\% with thick MSAF however other studies report Grade I thin MSAF $11.6 \%$ to $12.5 \%$ and thick meconium $87 \%$ to $90 \%$, with MAS in $21.2 \%$ [4, 5]. Risk factors associated with passage of meconium in utero include primiparity 50\% with MSAF and $45 \%$ in MAS, subsequently decreasing with increasing gravida also reported in other studies $[4,5]$.

Peak delivery with Grade II thick MSAF 38\% occurred at 39 weeks gestation followed by newborns with MAS $35 \%$ as well as Grade I thin MSAF 32\% also at 39 weeks gestation. However, peak births with clear liquor 27\% tookplace at 38 weeks gestation followed by $26.6 \%$ at 39 weeks gestation. Hence, later delivery at 39 weeks and 
beyond was associated with higher risk of MSAF and MAS signifying fetal distress with asphyxia injury as compared to 38 weeks gestation or less $[1,4,5]$.

MSAF and MAS was more common in young mothers with mean age 25 years, other studies reported MAS at 28 years, and mean birth weight varying between 2,855g in present study to 3,187g reported in other studies but with almost similar mean gestation at 38.2 to 38.9 weeks $[1,4,5]$. Factors associated with passage of meconium in utero include obstetric complications of placental insufficiency with PIH, non-reassuring foetal heart rate, foetal acidosis, emergency LSCS, meconium below cords and low Apgar score [2, 7, 11, 12].

Obstetric complications of PROM, though was commonest in pregnancies with clear liquor $40 \%$, however Grade II MSAF peaked 57\% followed by Grade I MSAF 35\%. PIH occurred mainly with MAS 57\% (n=4/7) with GDM at $43 \%$ as also Grade I MSAF at 52\% with least GDM of $13 \%$ to $21 \%$ in Grade II MSAF reported also in other studies $[4,5]$. Emergency LSCS was undertaken in 52\% of MAS cases, 51\% with Grade II thick MSAF and 47\% with Grade I thin MSAF. Other studies revealed 60.2\% LSCS in MSAF and 46.8\% in MAS [5]. In contrast, most births with clear liquor had a normal vaginal delivery between 38-39 weeks gestation, in younger mothers aged 20-24 years with second to third gravida and higher birth weight 2,500-3,499g.

Most parturient $33 \%$ had $\mathrm{AB}+$ ve blood group followed by $\mathrm{B}+\mathrm{ve} 31 \%$ and $\mathrm{O}+\mathrm{ve} 23 \%$, contrasts to general Caucasian and Asian population with majority $38 \% \mathrm{O}+\mathrm{ve}$ followed by $34 \% \mathrm{~A}+\mathrm{ve}$ in former and $32 \% \mathrm{~B}+\mathrm{ve}$ with least $3-7 \% \mathrm{AB}+\mathrm{ve}^{39}$. Could the unique predominance of $\mathrm{AB}+\mathrm{ve}$ mothers in pregnant women, be associated with fertility issues being universal recipients, as opposed to prevalence of $\mathrm{O}+\mathrm{ve}$ in general population being universal donors but have anti A and anti B of varying titers [26]. Mother's blood group type and Rh-ve factor is known to impact fetus and newborns.

The major goal of obstetric care is to prevent or reduce maternal, fetal and neonatal morbidity and mortality. In the present study, 207 cases of MSAF were associated with 84\%, asphyxiated newborns, OR 8.42 [CI 95\%] 5.1-14 being 30 times higher compared to newborns with clear liquor $(\mathrm{P}=0.0001)$ indicating that all cases with MSAF should be closely monitored with active management of labor and judicious intervention. The incidence of LSCS was high $42 \%$ with $60 \%$ being emergency LSCS, fetal distress being commonest indication reported in earlier study [27].The early identification of markers of fetal distress assumes importance as in the presence of hypoxia, plenty of meconium is passed and is dangerous, if aspirated. Management during prelabor with meconium staining includes ultrasonography which may reveal echogenic or particulate amniotic fluid [28] although; vernix may also have this appearance [29]. Amnioscopy first introduced by Saling [30], later replaced by modified contact endoscope and amniocentesis, however, amnioscopy is now used for the detection of meconium during antenatal period and in early labor. Amino-infusion is not recommended for routine treatment of suspected fetal compromise with MSAF as immediate delivery is a priority. Delivery attended by skilled birth attendant competent in neonatal intubation and tracheal suction recommended in Neonatal resuscitation program with intermittent positive pressure ventilation [31]. There is also limited evidence for the use of grading system for MSAF as its impact on neonatal outcome is on the volume aspirated that determines outcome of neonate more than concentration of MSAF, that inhibits fetal lung fluid reabsorption at birth and disturb the ability of the lungs in transition to extra uterine life continuous positive pressure (CPP) with oxygen facilitates fetal lung fluid reabsorption at birth, transiting smoothly from fetal to extra uterine life [13-15].

During the first and second stage of labor, foetal wellbeing is monitored by continuous electronic fetal heart rate tracing, the baseline fetal heart rate (FHR) best assessed between uterine contraction, by listening and counting for 60 seconds [10]. Features regarded as non-reassuring trace are baseline bradycardia $<110$ or tachycardia $>160 \mathrm{bpm}$, moderate to severe variable deceleration, late deceleration, loss of beat to beat variability and prolonged fetal bradycardia. Significantly higher non-reassuring foetal heart rate tracing was noted with MAS $(p<0.0001)$ [32, 33]. Decreased or absent beat to beat variability is an index of low fetal reserve or tolerance to hypoxic insults. In some circumstances of sudden, severe fetal hypoxia as in abruptio placenta or severe cord compression, exaggerated fetal movements is a warning sign as it is followed by loss of movement, necessitates prompt delivery, persistent FHR with tachycardia $>180 \mathrm{bpm}$ is an indication for quick intervention, while bradycardia $<100 \mathrm{bpm}$, if persistent indicates the terminal response of dying fetus, is an ominous pattern [32, 33].

Thus late decelerations, fetal bradycardia with MSAF becomes ominous if they occur along with deceased or absent beat to beat variability, however with several variable decelerations, cord prolapse or cord presentation should be ruled out, which is also an indication for caesarean section. However, there is no evidence that electronic fetal heart rate monitoring (EFM) without blood gas and acid base assessment reduces the risk of fetal or neonatal mortality or morbidity [32-34]. 
The classical signs or markers of fetal hypoxia are loss of, or decreased fetal movements, variations in fetal heart rate pattern, presence of meconium in amniotic fluid, presence of fetal moulding and decrease in fetal scalp blood $\mathrm{pH}$. A fetal scalp pH value below 7.20 is indicative of fetal acidosis, values between 7.20 and 7.25 are suspicious or pre acidotic, require further evaluation. If $<7.20$ or 7.17 immediate delivery by LSCS is recommended [31-33]. The best way to evaluate beat to beat variability is with fetal scalp $\mathrm{pH}$ determination by fetal pulse oximetry, which will improve decision-making in timing of delivery and may reduce the incidence of MSAF and MAS [34-36].

Biochemical parameters include fetal blood sampling for $\mathrm{pH}, \mathrm{pO}_{2}, \mathrm{pCO}_{2}$ and base deficits indicated in all high-risk patients with MSAF, fetal heart variation, with obstetric complications in pregnancy [33]. During contractions of normal labor, there is a decrease in utero-placental blood flow and subsequent increase in $\mathrm{pCO}_{2}$, decrease in $\mathrm{pO}_{2}$ and $\mathrm{pH}$. In severe variable decelerations pattern if, recurrent or un-correctable or the scalp $\mathrm{pH}$ shows fetal acidosis then the infant must be delivered by caesarean section [34, 35]

Increased moulding without descent of the head is a grave sign of fetal distress. Rapid development of moulding is also particularly serious. Stewart's scoring system is used to assess the degree of moulding assessed at least at two locations on the fetal head, i.e., parieto-occipital, parieto-parietal and if possible parieto frontal. It is scored in three grades. The moulding score is $0-6$. When there is $3+$ moulding at two sites, the fetus is in danger, if labour is allowed to proceed. Stewart as a sign of distress calling for early delivery considers excessive moulding an indication for LSCS.

The "Time Factor" between the discovery of the signs of fetal distress with fetal heart rate variation and delivery, is crucial as delivery should occur within 30 minutes with fetal distress. If delivered within 15 minutes of detection of fetal distress, the perinatal mortality was $0.5 \%$, if between $15-29$ minutes, then it was $3.65 \%$ and after 30 minutes it was 11 [25]. The fetal $\mathrm{pH}$ at this level will show no academia, and if the fetus is delivered within reasonable time, with immediate cord clamping and normal Apgar score will maintain umbilical and blood $\mathrm{pH}, \mathrm{pCO}_{2}$ partial pressure and base deficit values at optimum levels [25, 34].

At birth, intrapartum suctioning of the neonate mouth and pharynx on the perineum before delivery of shoulders is not recommended for routine practice [37,38], however, immediate new born care is cleaning mouth and nose of any visible meconium. Suctioning is not required if neonate is term and vigorous with good respiratory effort and muscle tone at birth, baby wiped dry and shifted to mothers' side after routine care. A vigorous preterm should be assessed and started on CPAP with low flow of oxygen at $4 \mathrm{~L} / \mathrm{min}$ and $\mathrm{FiO}_{2}<30 \%$ shifted to NICU for observation [38].

A non-vigorous neonate at birth should not be stimulated include drying but laryngoscopy with endotracheal intubations and tracheal suctioning done under direct vision, the meconium aspirator device attached to adapter of endotracheal tube and connected to a negative pressure source not exceeding $100 \mathrm{mmHg}$, occluding the side port and withdrawing over a few seconds. Repeated intubation is not recommended as it may cause further delay in resuscitation [37]. About one-third of infants with MAS may require intubation and mechanical ventilation [23, 31, 37]. Delivery room use of sustained inflation during neonatal resuscitation with CPPV and oxygen facilitates movement of meconium stained fetal lung fluid into interstitial to drain into lymphatic, aerating lungs, which triggers rapid complex physiological cardiovascular changes from fetal to neonatal circulation. CPPV is discontinued with regular breathing, $\mathrm{SpO}_{2}>90 \%$ and heart rate $>100 \mathrm{bpm}$ and plethysmograph tracing indicate how well heart is pumping oxygen through the body by showing pulsatile changes determined on pulse oximeter [13-15, 36].

After birth $\mathrm{SpO}_{2}$ is monitored for next 2 hours and assessed 2 hourly until 12 hours of age, normal temperature maintained between $36.5-37.4^{\circ}$, heart rate $120-160 \mathrm{bpm}$, respiratory rate $30-60 / \mathrm{min}$ and $\mathrm{SpO}_{2}>95 \%$. Neonate observed for features of respiratory distress such as chest wall movement, tone, color feeding and general well-being. Asymptomatic well babies with non-significant meconium stained liquor require observation at 1 and 2 hours of age and those with significant meconium staining of liquor require observation at 1 and 2 hours of age and then 2 hourly till 12 hours of age. Signs of respiratory distress are increase in respiratory rate, intercostal or subcostal retraction chest wall retraction, nasal flaring, head bobbing, cyanosis or grunting. Circulatory impairment monitored by heart rate, skin color, capillary refill checked. Investigations include CXR, FBC, ABG, blood cultures, CRP [37, 38].

Continuous positive airway pressure (CPAP) is a non-invasive respiratory support in preterm infants at birth with mild respiratory distress, early arterial and venous access required, nutrition maintained and humidified oxygen if required to keep pre-ductal oxygen saturation 95-98\% [38, 39]. In severe MAS with respiratory failure use of surfactant and inhaled nitric oxide (iNO) has led to decreased mortality and the need for extracorporeal membrane oxygenation (ECMO) [40, 41]. However, aggressive management during and immediately after delivery does not always prevent fatal meconium aspiration syndrome hence prevention is better with quick intervention and delivery. 


\section{Conclusion}

The identification of high risk factors with close supervision, active management of labor, and judicious obstetric intervention is essential to improving fetal and neonatal outcome as $84 \%$ of MSAF were associated with birth asphyxia, resulted in emergency LSCS in over $50 \%$ with MSAF and MAS, while most new-borns with clear liquor were delivered normally at 38 weeks with only one-fourth by emergency section. MSAF incidence was $7.8 \%$ and MAS was $1.8 \%$, but it constituted 23\% of MSAF parturient. Since fetal asphyxia may result in fatal MAS, hence prevention pays as peak MSAF and MAS births took place at 39 weeks gestation, indicates that early delivery preferably at 38 weeks in high risk cases would preclude to birth asphyxia and its consequences of MSAF and MAS, thereby improving fetal and neonatal outcome.

\section{References}

[1] Arun, H., Nayak, A., and Dalal R. (1991). Meconium staining of amniotic fluid- Significance and fetal outcome. Indian Jl OBG Aug 41(4): 480-83.

[2] Cleary, G. M. and Wisell, T. E. (1998). MSAF and MAS an update Pediatrics Clinics of North America. Vol. 45, No. 3 pp. 511-529.

[3] Bardley, A. Yoder, E. A. Kirsch, William H. B., and Michael G. C. (2002). Changing obstetric practices associated with decreasing incidence of meconium aspiration syndrome. Am J Obset Gynecol, 99(5): 731-39.

[4] Addisu, D., Asres, A., Gedefav, G. and Asmer, S. et al. (2018). Prevalence of Meconium stained Amniotic fluid and factors among women who gave birth at term in Felege Hivot Comprehensive specialized hospital, North West Ethiopia: A facility based cross-sectional study. BMC. Pregnancy and Childbirth, 18: 429.

[5] Khazardoost, Hanloushzadeh, S., Khooshideh, M., and Borna, S. (2007). Risk factors for Meconium aspiration in Meconium stained Amniotic fluid. J Obstet Gynecol 27(6): 577-579.

[6] Miller, F. C., D. A. Sachs, S. Y. Yeh, et al. (1975). Significance of meconium during labor. Am J Obstet Gynecol 122(5): 573-580.

[7] Katz, L. V. and Bowes W. A. (1992). Meconium aspiration syndrome, reflection on a musky subject. Am. J. Obstet \& Gynaecol 166: 171-183.

[8] Gabbe, S. G., Ettinger, B. B., Freeman, R. K., and Martin, C. B. (1976). Umbilical cord compression associated with amniotomy: laboratory observations. Am J Obstet Gynecol, 126(3): 353-355.

[9] Abramovici, H., Brandus, T. H., Fuchs, K., and Timor-Tritsch, I. (1974). Meconium during delivery, a sign of compensated fetal distress. Am J. Obset \& Gynaecol 118(2): 251-255.

[10] Hon, E. H., Brad, Field, A. M. and Hessow. (1961). The electronic evaluation of the fetal heart rate. Am. J. Obstet Gynecol, (82): 291

[11] Socol, M. L., Garcia, P. M., and Riter, S. (1994). Depressed Apgar scores, Acid-base status, and neurologic outcome. Am J Obset Gynoco, 170(4): 991.

[12] Starks, G. C. (1980). Correlation of meconium stained amniotic fluid, early intrapartum fetal $\mathrm{pH}$ an Apgar scores as predictors of perinatal outcome. Journal of Obstet \& Gynaecol 56: 604.

[13] Jovanovic, R. and Nguyen, H. T. (1989). Experimental meconium aspiration in guinea pigs. Obstet \& Gynecolvol, 73(4): 652-656.

[14] Keszler, Martin. (2015). "Sustained inflation during neonatal resuscitation” Current Opinion in Pediatrics.

[15] Chua, B. A., Chan, L., Kindker, P. M., and Perks, A. M. (2000). "The association between meconium and the production and reabsorption of lung liquid and lactate loss by in vitro lungs from fetal guinea pigs”. Am J Obstet \& Gynecol 183(1): 235-244.

[16] Gooding, C. A., Gregory, G. A., and Taber, P. (1971). An experimental model for the study of meconium aspiration of the new born. Radiology, 100(1): 137.

[17] Florman, A. l. and Tuebner, D. (1986). Enhancement of bacterial growth in amniotic fluid by meconium. J Pediatr, 74(1): 111.

[18] Romero, R., Hanaoka, S., and Mazor, M. et al. (1991). Meconium stained amniotic fluid: A risk factor for microbial invasion of the amniotic cavity. Am. J. Obset\& Gynaecol 16(3): 859-862.

[19] Clark, P. and Duff, P. (1995). Inhibition of neutrophil oxidative burst and phagocytosis by meconium. Am J Obstet Gynecol. 173(4): 1301.

[20] Murphy, J. D., Rabinovitch, M., and Goldsteen, J. D. (1991). The structural basis of persistent pulmonary hypertension of the newborn infant. J Pediatr, 98(6): 962-967.

[21] Periman, I., Moore, G. W., and Hutchins, G. M. (1989). The pulmonary vasculature in meconium aspiration. Human pathology 20(7): 701-706.

[22] Tyler, D., Murphy, J., and Chenny, F. W. (1978). Mechanical and chemical damage to lung tissue caused by meconium aspiration. Jr. Pediatrics, 62: 454-59. 
[23] Vidyasagar, Yeh, T. F., and Harris, V. (1975). Assisted ventilation in infants with meconium aspiration syndrome. Pediatrics J., 56: 208.

[24] Ostrea, Jr. E. M. and Naqvi, M. (1982). The influence of gestational age on the ability of the fetus to pass meconium in utero: clinical implications. Acta Obstetricia et Gynecological Scandinavica, 61(3), pp. 275-277.

[25] Fenton, A. N. and Steer, C. M. (1962). Meconium in utero, a sign of fetal maturity. Am. J. Obstet \& Gynaecol, $83: 354$.

[26] Piui, T. What I the most common blood type? zmescience.com/science/m

[27] Grace Lalana Christopher. (2018). The effect of biological factors on Birth weight and Gestation in South Indian Newborns. J Pediatr Neurol Med., 3(1), pp. 97-103. DOI: 104172/2472-100X1000130.

[28] Benacerrf, B. R., Gatter, M. A., and Ginsburgh, F. (1984). Ultrasound diagnosis of meconium-stained amniotic fluid. Am J obset gynecol, 149(5): 570-572.

[29] Hill, L. M. and Breckle, R. (1998). Vernix in amniotic fluid: Sonography detection. Radiology, 158(1): 80.

[30] Saling, E. W. (1966). Amnioscopy. J. of Clinical Obstet \& Gynaecol 9: 472.

[31] Wyckoff, M. H., Aziz, K., Esobedo, M. B., Kapadia, VS et al. (2015). Part 13: neonatal resuscitation: 2015 American Heart Association Guidelines Update for Cardiopulmonary Resuscitation and Emergency Cardiovascular care. Circulation, 132 (suppl 2): S543-S560.

[32] Kubli. (1988). Significance of second stage fetal heart rate abnormalities. Am J. Obset\& Gynaecol 60: 330-32.

[33] Alfirev, Z., Devane, D., and Gyte, G. M. (2006). Continuous Cardiotocography (CTG) as a form of electronic fetal monitoring (EFM) for fetal assessment during labor. Cochrane Database of Systematic Reviews No. 3; Article ID CD006066, 2006.

[34] Kuhnert, M., Seelbach-Goebel, B., and Butterwegge, M. (1998). Predictive agreement between the fetal arterial oxygen saturation and fetal scalp pH: results of the German multicenter study. Am J Obstet Gynecol, 178(2): 330-335.

[35] Low, J. A. and Piercy, W. N. (1981). The prediction of intrapartum fetal metabolic acidosis by fetal heart rate monitoring. Am J Obstet Gynecol, 139(3), Feb, pp. 299-305.

[36] Nicholas, Johnson. (1994). "Fetal Pulse Oximetry: A new method of monitoring the Fetus”. The Australian and New Zealand Journal of Obstetrics and Gynecology, 34(4): 428-432.

[37] Velaphi, S. and Vidyasagar, D. (2006). Intrapartum and post-delivery management of infants born to mothers with MSAF: evidence based recommendation. Clinics in Perinatology, 33(1): 29-42.

[38] Wiswell, T. E. (2008). Delivery room management of the meconium stained newborn. Journal of Perinatology, 28(suppl. 3): S19-26.

[39] Schmolzer, G., M., Kumar, M., and Pichler, G. et al. (2013). Non-invasive versus invasive respiratory support in preterm infants at birth: systematic review and meta-analysis. BMJ. 2013; 347 doi: https:/doi.org/10.1136/bmj.f5980 (published 17 October 2013).

[40] Kinella, J. P., Truog, W. E., and Walsh, W. F. et al. (1997). Randomized multicenter trial of inhaled nitric oxide and high frequency oscillatory ventilation in severe, persistent pulmonary hypertension of the newborn. J Pediatr., 131(1), part 1, pp. 55-62.

[41] Dargaville, P. A., Copnell, B., and Mills, J. F. et al. (2011). Randomized controlled trial of lung lavage with dilute surfactant for meconium aspiration syndrome. J Pediatr, 158(3), pp. 383-389. 